\title{
Muscle Strength and Power: Primary Outcome Measures to Assess Cold Water Immersion Efficacy After Exercise With a Strong Strength or Power Component
}

\author{
Angus Lindsay ${ }^{1 *}$ and Jonathan M. Peake ${ }^{2,3}$ \\ ${ }^{1}$ Institute for Physical Activity and Nutrition, School of Exercise and Nutrition Sciences, Deakin University, Geelong, VIC, \\ Australia, ${ }^{2}$ School of Biomedical Sciences, Queensland University of Technology, Brisbane, QLD, Australia, ${ }^{3}$ Sport \\ Performance Knowledge and Innovation Excellence, Queensland Academy of Sport, Brisbane, QLD, Australia
}

Keywords: cryotherapy, recovery, performance, muscle damage, exercise, excitation contraction coupling

\section{INTRODUCTION}

OPEN ACCESS

Edited by:

Mohammed Ihsan,

National University of

Singapore, Singapore

Reviewed by: James Robert Broatch,

Victoria University, Australia

Aaron Petersen,

Victoria University, Australia

${ }^{*}$ Correspondence:

Angus Lindsay

a.lindsay@deakin.edu.au

Specialty section:

This article was submitted to

Elite Sports and Performance

Enhancement,

a section of the journal

Frontiers in Sports and Active Living

Received: 19 January 2021

Accepted: 18 May 2021

Published: 14 June 2021

Citation:

Lindsay A and Peake JM (2021)

Muscle Strength and Power: Primary

Outcome Measures to Assess Cold

Water Immersion Efficacy After

Exercise With a Strong Strength or

Power Component.

Front. Sports Act. Living 3:655975.

doi: 10.3389/fspor.2021.655975
Recovery from exercise-induced muscle damage, fatigue or stress is critical for restoration of exercise performance. In most exercise activities or sports, performance is regulated through the physiological capacity of muscle. Therefore, athletes, coaches, and scientists have continued to explore post-exercise recovery modalities that focus on expediting muscle functional recovery. Several post-exercise recovery strategies have been developed, tested, and used in amateur and professional athletes to expedite muscle functional recovery. A cost-effective and well-researched practice to achieve this goal is cold water immersion (CWI). CWI requires submersion of a limb or the whole body in cold water of a specified temperature (usually $<15^{\circ} \mathrm{C}$ ) for a specified duration immediately post-exercise, or over several succeeding days. However, the equivocal findings on the efficacy of CWI and the extensive number of outcome variables has made it challenging to interpret and correctly implement this intervention. In this short opinion piece, we briefly review CWI research and the challenges that practitioners and athletes face when deciding whether to use CWI as a post-exercise recovery intervention. We then discuss why muscle strength and/or power should be considered the primary outcome variable in CWI research with a strong strength and power component, and why excitation-contraction coupling and/or rate of force development assessment is necessary to evaluate strength/power-specific changes. Finally, we present systematic evidence that there is a dearth of strength measurements in CWI research, which could be limiting our understanding of this post-exercise recovery strategy.

\section{A BRIEF HISTORY OF CWI RESEARCH}

Some of the first evidence that a single CWI application might be beneficial for recovery from muscle damage or injury was provided by Hayden (Hayden, 1964) and Hocutt et al. (1982). Both studies showed that CWI expedited return-to-duty in soldiers after injury, or return to full activity after ankle sprain, respectively. In contrast, Matsen et al. and Marek et al. showed that application of cold water to an injury significantly increased oedema (Matsen et al., 1975; Marek et al., 1979). Subsequent research about the effects of CWI has also produced some contrasting findings. For example, CWI immediately following blunt trauma to skeletal muscle of rats significantly reduced oedema formation (Dolan et al., 1997). By contrast, CWI following eccentric contractions did not affect muscle soreness or strength in humans (Eston and Peters, 1999). More recently, Naderi et al. 
showed that CWI did not attenuate a loss in muscle strength following a single bout of strength training (Naderi et al., 2021), whereas Kodejška et al. (2018) demonstrated that CWI increased the force time integral in handgrip performance compared with passive recovery in rock climbers. Differences in CWI study outcomes could be associated with different modes of exercise, methods or timing of applying CWI and approaches to assessing muscle damage.

Contradictory findings related to repeated CWI applications have also been reported and confirmed in a recent systematic review and meta-analysis (Malta et al., 2021). Fu et al. (1997) showed that when CWI was regularly applied to rats after exercise training, it caused advanced ultrastructural damage to myofibrils. Several human studies have also shown negative adaptive effects of repeated CWI applications after resistance training (Fröhlich et al., 2014; Roberts et al., 2015; Yamane et al., 2015; Fyfe et al., 2019; Poppendieck et al., 2020). However, Lindsay et al. (2016) showed that repeated CWI applied to mixed martial artists during a training camp attenuated the inflammatory response, but did not affect measures of performance. Repeated CWI applied after high intensity interval training (HIIT) or a combination of HIIT with low-moderate intensity aerobic exercise also does not influence indices of performance or muscle cellular signaling (Halson et al., 2014; Aguiar et al., 2016; Christiansen et al., 2018). Although these studies represent only a small proportion of published CWI research, they do demonstrate the complexity in understanding the value of this recovery intervention.

\section{INTERPRETING CWI RESEARCH}

Regardless of the inconsistencies in CWI study outcomes, anecdotal evidence suggests that professional athletes from various sports use CWI as a post-exercise recovery strategy. The reasons behind this persistent practice are uncertain, but may reflect a disconnect between the scientific findings of the studies, and how coaches and athletes interpret these findings. The practitioners' guide to determining if, and when, to implement CWI is confounded by the vast performance, biochemical, and qualitative analyses that have been used to evaluate its efficacy. Other than the variability in CWI protocols (which can range from 4 to $15^{\circ} \mathrm{C}, 5$ to $30 \mathrm{~min}$ durations, 1 to 10 applications, immediate to delayed submersion) and level of exercise intensity, participant sex and training status, the outcome variables of interest provide an added level of complexity in CWI study comparisons. First, a practitioner or self-coached athlete without in-depth scientific knowledge of biological processes, may not be able to interpret correctly the results of CWI studies that focus on indices of inflammation, gene expression or rates of protein synthesis. Second, drawing comparisons between performance and biochemical or molecular variables could be challenging for a non-scientist. Physiological performance analysis offers a direct and interpretable option for practitioners that is training-specific. We therefore propose that consistently measuring maximal muscular strength and/or power [product of load lifted and angular displacement (distance load moved) divided by time spent moving the load (Sapega and Drillings, 1983; Winter et al., 2016; Horta-Gim et al., 2021)] will provide the exercise community with a more appropriate understanding of whether CWI enhances recovery from exercise-induced damage or fatigue, and improves the performance and work capacity of athletes. From our perspective as exercise physiologists, maintenance of muscular strength and power, irrespective of any changes in muscle ultrastructural integrity, will likely benefit overall physical performance.

\section{MUSCLE STRENGTH/POWER AND MECHANISMS OF STRENGTH/POWER LOSS}

Muscle strength and power outcomes is a multi-faceted coordination of electrical and chemical events, together with interactions between structural components of muscle tissue. Strength and power are measured using the 1-repetiton maximum (actual or estimated), or with force transducers or plates associated with lab-based dynamometers that measure absolute torque production and rate of force development. Loss of muscle strength associated with eccentric contractions (which lengthen the muscle during simultaneous force production) can be primarily attributed to excitation-contraction uncoupling, and to a lesser extent, loss of contractile protein and structural damage (Warren et al., 2001, 2002). Therefore, post-exercise recovery interventions should target the processes of excitationcontraction coupling to accelerate recovery from eccentric contraction-biased exercise. The triad of skeletal muscle is the site of excitation-contraction uncoupling following eccentric contraction-induced strength loss. More specifically, it is the voltage-sensitive dihydropyridine receptors (DHPR) located in the T-tubules and the ryanodine receptor (RyR) calcium release channel of the sarcoplasmic reticulum (Ingalls et al., 1998; Warren et al., 2001, 2002; Corona et al., 2010; Baumann et al., 2014). The sensitivity of both the DHPR and RyR are not affected by eccentric contraction-induced strength loss (Ingalls et al., 2004a). However, the expression of proteins that associate with the DHPR and RyR to modulate cross-talk and calcium release is significantly decreased (Corona et al., 2010; Baumann et al., 2014). Thus, assessing the effectiveness of CWI for restoring muscle strength could include molecular measurement of the DHPR, RyR, junctophilin, FKPB12, calmodulin, or calsequestrin (proteins associated with the triad of muscle fibers and known to interact with channels and receptors regulating skeletal muscle calcium kinetics). Because cold acclimation can influence calcium handling/kinetics of skeletal muscle and improve indices of muscle performance (Bruton et al., 2010), additional calcium measurements following CWI could supplement analyses of excitation-contraction coupling. However, we do acknowledge that such measurements of proteins following CWI would require time course evaluation, multiple muscle biopsies that would complicate human studies with respect to recruitment and full participation, and confound interpretations of findings by non-scientists. 
Researchers can indirectly assess excitation-contraction uncoupling in vivo by comparing the low-frequency to highfrequency torque loss prior to and following CWI. The greater reduction in low-frequency torque compared with highfrequency torque indicates excitation-contraction uncoupling (Edwards et al., 1977; Jones et al., 1982; Ingalls et al., 2004b). Cheng et al. (2017) showed that in isolated single muscle fibers of mice, cold application following fatiguing contractions dampened submaximal force without altering maximal force during recovery. In fact, the ratio of submaximal to maximal force was lowest with the coldest temperature, suggesting greater excitation-contraction uncoupling with colder applications. Additionally, we acknowledge that force-generating capacity during rapid, dynamic movements is also relevant to athletic performance and may represent a more sensitive measure to detect changes in neuromuscular function. Rate of force development can generally be determined by measuring the change in peak force divided by a change in time (maximal rate of contraction to accommodate for inter-individual variability in peak force development time) using lab-based force transducers and associated software. Central nervous system (CNS) fatigue likely also influences recovery of muscle strength and/or power following a single or repeated applications of strenuous exercise (Peiffer et al., 2009). Therefore, measuring CNS fatigue would also improve the assessment of muscle function recovery. Noninvasive CNS assessments could use an interpolated twitch during a maximal voluntary contraction (Allen et al., 1995) but would require the use of stimulation units. Collectively, more research of this nature will help to improve understanding of how CWI influences muscle function.

Loss of muscle strength and/or power can also be caused by fatiguing contractions (i.e., short-term strength and/or power loss caused primarily by energy depletion, and/or short-term "reversible" decrements in excitation contraction coupling) or blunt force trauma-the latter of which causes damage to structural and force-generating proteins of the muscle. Therefore, restoration of muscle strength and/or power by CWI would ideally need to affect several components of excitation contraction-coupling, synthesis of essential proteins, and restoration of the muscle architecture. CWI is thought to expedite recovery from exercise by lowering skin, intramuscular and body temperature, cardiovascular strain, blood flow and increasing metabolism, blood pressure and heart rate (Bleakley and Davison, 2010b; Ihsan et al., 2016). Although CWI does not influence glycogen resynthesis rates after exhaustive exercise in humans (Gregson et al., 2013), other cryotherapy applications can reduce inflammatory cell infiltration after soft tissue injuries in animal studies (Bleakley and Davison, 2010a) and CWI can lower inflammatory biomarkers after contact sport (Lindsay et al., 2017) and resistance exercise in humans (Missau et al., 2018). However, there are equivocal findings that CWI does not affect muscle-specific or circulating inflammatory biomarkers after resistance exercise (Peake et al., 2017a), repeated sprints (White et al., 2014) or volleyball training (De Freitas et al., 2019) in humans. This variation may be attributed to the level of muscle damage imposed by the initial exercise. The first wave of responders to sites of muscle damage (strength and/or power loss) includes granulocytes, and mononucleated cells such as macrophages, eosinophils and monocytes. Considering that cold-stress limits mononuclear cell activity (Lindsay et al., 2016; Reynés et al., 2019), and inflammation is integral to muscle repair and regeneration (Peake et al., 2017b), it follows that CWI may in fact delay the sequence of events involved in muscle repair (Tidball, 2011) and the recovery of muscle strength and/or power. Additionally, CWI may slow recovery from structural protein damage, because protein synthesis, ribosomal biogenesis and anabolic signaling are temperature-dependent (Roberts et al., 2015; Figueiredo et al., 2016; Fuchs et al., 2020). Overall, the mechanisms by which CWI may affect recovery of muscle strength and/or power have not definitively been determined.

Ensuring muscle strength and power measurements are considered as a primary outcome measure for CWI studies investigating forms of exercise in which recovery of strength/power is important (independent of inflammatory status or the ultrastructural integrity of the muscle) is critical. This is because even muscle that is severely structurally compromised, with a steady state of inflammation and heightened sensitivity to exercise-induced loss of sarcolemmal excitability, can produce strength and power. For example, skeletal muscle from dystrophin-deficient mice, a model of Duchenne muscular dystrophy, undergoes continuous cycles of degeneration and regeneration, inflammation, exercise-induced loss of sarcolemmal excitability and replacement of muscle with adipose and fibrotic tissue (Tanabe et al., 1986; Baumann et al., 2020). Functional analyses indicate that absolute strength and rate of force development during a twitch and tetanic contraction of these dystrophin-deficient muscles in mice is not different to healthy skeletal muscles (Lindsay et al., 2019). However, although inflammatory status and skeletal muscle integrity might not affect muscle strength and/or power in a diseased state, it may predispose muscle of healthy individuals to greater levels of exercise-induced stress that could, in turn, lead to poorer long-term performance or extended recovery periods.

\section{BRIEF SYSTEMATIC REVIEW - CWI AND MUSCLE STRENGTH}

Despite the variation in outcome variables and advancements in muscle strength and power assessment technologies for CWI research in humans, relatively few studies have included the measurement of muscle strength and power as a measure of the effectiveness of CWI. A literature search in PubMed identified a total of 427 peer-reviewed studies on "cold water immersion" AND "muscle" (01/12/2020). Of these 427 studies, 31 (7\%) measured muscle strength prior to and following exercise and CWI. Of the 31 studies that measured strength prior to and following an intervention, 14 studies showed positive effects for CWI over a passive or active recovery modality on strength and/or power variables, six studies showed that CWI was detrimental to muscle strength and/or power, and 11 studies showed no effect. Twenty-one of the 31 studies completed only a single application of CWI, whereas 10 studies completed two or more applications of CWI. Overall, our literature search of CWI 
and muscle strength measurements provides conflicting evidence that CWI has beneficial effects for muscle strength variables.

\section{CONCLUSION}

The efficacy of CWI has been tested and studied for decades, with large variation in outcomes. Although outcome measures remain relatively constant, the difficulty in assessing CWI as a strategy for post-exercise recovery is associated with the variability in the intervention itself. While investigating CWI protocol variables does provide additional information, it somewhat contributes to the level of confusion accompanying this modality for amateur and professional athletes. Therefore, we re-iterate that independent of the CWI protocol used in a study setting, that measures of absolute or relative muscle strength and/or power should be the primary measurement. This approach will at least

\section{REFERENCES}

Aguiar, P. F., Magalhães, S. M., Fonseca, I. A. T., da Costa Santos, V. B., de Matos, M. A., Peixoto, M. F. D., et al. (2016). Post-exercise cold water immersion does not alter high intensity interval training-induced exercise performance and Hsp72 responses, but enhances mitochondrial markers. Cell Stress Chaperones 21, 793-804. doi: 10.1007/s12192-016-0704-6

Allen, G. M., Gandevia, S. C., and McKenzie, D. K. (1995). Reliability of measurements of muscle strength and voluntary activation using twitch interpolation. Muscle Nerve 18, 593-600. doi: 10.1002/mus.880180605

Baumann, C. W., Rogers, R. G., Gahlot, N., and Ingalls, C. P. (2014). Eccentric contractions disrupt FKBP12 content in mouse skeletal muscle. Physiol. Rep. 2:e12081. doi: 10.14814/phy2.12081

Baumann, C. W., Warren, G. L., and Lowe, D. A. (2020). Plasmalemma function is rapidly restored in Mdx muscle after eccentric contractions. Med. Sci. Sports Exerc. 52, 354-361. doi: 10.1249/MSS.0000000000002126

Bleakley, C. M., and Davison, G. W. (2010a). Cryotherapy and inflammation: evidence beyond the cardinal signs. Phys. Ther. Rev. 15, 430-435. doi: 10.1179/1743288X10Y.0000000014

Bleakley, C. M., and Davison, G. W. (2010b). What is the biochemical and physiological rationale for using cold-water immersion in sports recovery? A systematic review. Br. J. Sports Med. 44, 179-187. doi: 10.1136/bjsm.2009.065565

Bruton, J. D., Aydin, J., Yamada, T., Shabalina, I. G., Ivarsson, N., Zhang, S.-J., et al. (2010). Increased fatigue resistance linked to $\mathrm{Ca}^{2+}$-stimulated mitochondrial biogenesis in muscle fibres of cold-acclimated mice. J. Physiol. 588, 4275-4288. doi: 10.1113/jphysiol.2010.198598

Cheng, A. J., Willis, S. J., Zinner, C., Chaillou, T., Ivarsson, N., Ørtenblad, N., et al. (2017). Post-exercise recovery of contractile function and endurance in humans and mice is accelerated by heating and slowed by cooling skeletal muscle. J. Physiol. 595, 7413-7426. doi: 10.1113/JP274870

Christiansen, D., Bishop, D. J., Broatch, J. R., Bangsbo, J., McKenna, M. J., and Murphy, R. M. (2018). Cold-water immersion after training sessions: effects on fiber type-specific adaptations in muscle $\mathrm{K}$ transport proteins to sprint-interval training in men. J. Appl. Physiol. 125, 429-444. doi: 10.1152/japplphysiol.00259.2018

Corona, B. T., Balog, E. M., Doyle, J. A., Rupp, J. C., Luke, R. C., and Ingalls, C. P. (2010). Junctophilin damage contributes to early strength deficits and EC coupling failure after eccentric contractions. Am. J. Physiol. Physiol. 298, C365-C376. doi: 10.1152/ajpcell.00365.2009

De Freitas, V. H., Ramos, S. P., Bara-Filho, M. G., Freitas, D. G. S., Coimbra, D. R., Cecchini, R., et al. (2019). Effect of cold water immersion performed on successive days on physical performance, muscle damage, and inflammatory, hormonal, and oxidative stress markers in volleyball players. J. Strength Cond. Res. 33, 502-513. doi: 10.1519/JSC.0000000000001884 offer scientists, athletes and coaches a comparison among CWI studies in the outcome variable that is relatively easy to interpret, and matters most to athletic performance.

\section{AUTHOR CONTRIBUTIONS}

AL conceived and wrote the opinion. JP conceived and critically reviewed the opinion. All authors contributed to the article and approved the submitted version.

\section{FUNDING}

AL is supported by an Alfred Deakin Postdoctoral Research Fellowship. JP is supported by Sport Performance and Knowledge Innovation Excellence at the Queensland Academy of Sport, Brisbane, Australia.

Dolan, M., Thornton, R., Fish, D., and Mendel, F. (1997). Effects of cold water immersion on edema formation after blunt injury to the hind limbs of rats. $J$. Athl. Train. 32, 233-237.

Edwards, R. H., Hill, D. K., Jones, D. A., and Merton, P. A. (1977). Fatigue of long duration in human skeletal muscle after exercise. J. Physiol. 272, 769-778. doi: 10.1113/jphysiol.1977.sp012072

Eston, R., and Peters, D. (1999). Effects of cold water immersion on the symptoms of exercise-induced muscle damage. J. Sports Sci. 17, 231-238. doi: 10.1080/026404199366136

Figueiredo, V. C., Roberts, L. A., Markworth, J. F., Barnett, M. P. G., Coombes, J. S., Raastad, T., et al. (2016). Impact of resistance exercise on ribosome biogenesis is acutely regulated by post-exercise recovery strategies. Physiol. Rep. 4:12670. doi: 10.14814 /phy2.12670

Fröhlich, M., Faude, O., Klein, M., Pieter, A., Emrich, E., and Meyer, T. (2014). Strength training adaptations after cold-water immersion. J. Strength Cond. Res. 28, 2628-2633. doi: 10.1519/JSC.0000000000000434

Fu, F. H., Cen, H.-W., and Eston, R. G. (1997). The effects of cryotherapy on muscle damage in rats subjected to endurance training. Scand. J. Med. Sci. Sports 7, 358-362. doi: 10.1111/j.1600-0838.1997.tb00167.x

Fuchs, C. J., Kouw, I. W. K., Churchward-Venne, T. A., Smeets, J. S. J., Senden, J. M., van Marken Lichtenbelt, W. D., et al. (2020). Postexercise cooling impairs muscle protein synthesis rates in recreational athletes. J. Physiol. 598, 755-772. doi: 10.1113/JP278996

Fyfe, J. J., Broatch, J. R., Trewin, A. J., Hanson, E. D., Argus, C. K., Garnham, A. P., et al. (2019). Cold water immersion attenuates anabolic signaling and skeletal muscle fiber hypertrophy, but not strength gain, following whole-body resistance training. J. Appl. Physiol. 127, 1403-1418. doi: 10.1152/japplphysiol.00127.2019

Gregson, W., Allan, R., Holden, S., Phibbs, P., Doran, D., Campbell, I., et al. (2013). Postexercise cold-water immersion does not attenuate muscle glycogen resynthesis. Med. Sci. Sports Exerc. 45, 1174-1181. doi: 10.1249/MSS.0b013e3182814462

Halson, S. L., Bartram, J., West, N., Stephens, J., Argus, C. K., Driller, M. W., et al. (2014). Does hydrotherapy help or hinder adaptation to training in competitive cyclists. Med. Sci. Sports Exerc. 46, 1631-1639. doi: 10.1249/MSS.0000000000000268

Hayden, C. A. (1964). Cryokinetics in an early treatment program. Phys. Ther. 44, 990-993. doi: 10.1093/ptj/44.11.990

Hocutt, J. E., Jaffe, R., Rylander, C. R., and Beebe, J. K. (1982). Cryotherapy in ankle sprains. Am. J. Sports Med. 10, 316-319. doi: 10.1177/036354658201000512

Horta-Gim, M., Romero-Pérez, E., Medina-Pérez, C., Tánori-Tapia, J., NúñezOthón, G., Novo, A., et al. (2021). Test-retest and minimal detectable change in the assessment of muscle strength and muscle power in upper and lower extremity exercises in 9- to 14-year-old children. Appl. Sci. 11:2204. doi: 10.3390/app11052204 
Ihsan, M., Watson, G., and Abbiss, C. R. (2016). What are the physiological mechanisms for post-exercise cold water immersion in the recovery from prolonged endurance and intermittent exercise? Sport. Med. 46, 1095-1109. doi: 10.1007/s40279-016-0483-3

Ingalls, C. P., Warren, G. L., Williams, J. H., Ward, C. W., and Armstrong, R. B. (1998). E-C coupling failure in mouse EDL muscle after in vivo eccentric contractions. J. Appl. Physiol. 85, 58-67. doi: 10.1152/jappl.1998.85.1.58

Ingalls, C. P., Warren, G. L., Zhang, J.-Z., Hamilton, S. L., and Armstrong, R. B. (2004a). Dihydropyridine and ryanodine receptor binding after eccentric contractions in mouse skeletal muscle. J. Appl. Physiol. 96, 1619-1625. doi: 10.1152/japplphysiol.00084.2003

Ingalls, C. P., Wenke, J. C., Nofal, T., and Armstrong, R. B. (2004b). Adaptation to lengthening contraction-induced injury in mouse muscle. J. Appl. Physiol. 97, 1067-1076. doi: 10.1152/japplphysiol.01058.2003

Jones, D. A., Howell, S., Roussos, C., and Edwards, R. H. (1982). Low-frequency fatigue in isolated skeletal muscles and the effects of methylxanthines. Clin. Sci. (Lond). 63, 161-167. doi: 10.1042/cs0630161

Kodejška, J., Baláš, J., and Draper, N. (2018). Effect of cold-water immersion on handgrip performance in rock climbers. Int. J. Sports Physiol. Perform. 13, 1097-1099. doi: 10.1123/ijspp.2018-0012

Lindsay, A., Carr, S., Cross, S., Petersen, C., Lewis, J. G., and Gieseg, S. P. (2017). The physiological response to cold-water immersion following a mixed martial arts training session. Appl. Physiol. Nutr. Metab. 42, 529-536. doi: 10.1139/apnm-2016-0582

Lindsay, A., Othman, M. I., Prebble, H., Davies, S., and Gieseg, S. P. (2016). Repetitive cryotherapy attenuates the in vitro and in vivo mononuclear cell activation response. Exp. Physiol. 101, 851-865. doi: 10.1113/EP085795

Lindsay, A., Southern, W. M., McCourt, P. M., Larson, A. A., Hodges, J. S., Lowe, D. A., et al. (2019). Variable cytoplasmic actin expression impacts the sensitivity of different dystrophin-deficient mdx skeletal muscle to eccentric contraction. FEBS J. 286, 2562-2576. doi: 10.1111/febs.14831

Malta, E. S., Dutra, Y. M., Broatch, J. R., Bishop, D. J., and Zagatto, A. M. (2021). The effects of regular cold-water immersion use on training-induced changes in strength and endurance performance: a systematic review with meta-analysis. Sport. Med. 51, 161-174. doi: 10.1007/s40279-020-01362-0

Marek, J., Jezdinsky, J., and Ochonsky, P. (1979). Effects of local cold and heat therapy on traumatic edema of the rat hind paw. III. The effect of heat radiation on the course of traumatic edema. Schmidt al. Z Rheumatol. 38, 391-404.

Matsen, F. A., Questad, K., and Matsen, A. L. (1975). The effect of local cooling on postfracture swelling. Clin. Orthop. Relat. Res. 109, 201-206. doi: 10.1097/00003086-197506000-00029

Missau, E., Teixeira, A. O., Franco, O. S., Martins, C. N., Paulitsch, F. S., Peres, W., et al. (2018). Cold water immersion and inflammatory response after resistance exercises. Rev. Bras. Med. do Esporte 24, 372-376. doi: 10.1590/1517-869220182405182913

Naderi, A., Aminian-Far, A., Gholami, F., Mousavi, S. H., Saghari, M., and Howatson, G. (2021). Massage enhances recovery following exercise-induced muscle damage in older adults. Scand. J. Med. Sci. Sports. 31, 623-632. doi: $10.1111 /$ sms. 13883

Peake, J. M., Neubauer, O., Della Gatta, P. A., and Nosaka, K. (2017a). Muscle damage and inflammation during recovery from exercise. J. Appl. Physiol. 122, 559-570. doi: 10.1152/japplphysiol.00971.2016

Peake, J. M., Roberts, L. A., Figueiredo, V. C., Egner, I., Krog, S., Aas, S. N., et al. (2017b). The effects of cold water immersion and active recovery on inflammation and cell stress responses in human skeletal muscle after resistance exercise. J. Physiol. 595, 695-711. doi: 10.1113/JP272881

Peiffer, J. J., Abbiss, C. R., Nosaka, K., Peake, J. M., and Laursen, P. B. (2009). Effect of cold water immersion after exercise in the heat on muscle function, body temperatures, and vessel diameter. J. Sci. Med. Sport 12, 91-96. doi: 10.1016/j.jsams.2007.10.011

Poppendieck, W., Wegmann, M., Hecksteden, A., Darup, A., Schimpchen, J., Skorski, S., et al. (2020). Does cold-water immersion after strength training attenuate training adaptation? Int. J. Sports Physiol. Perform. 16:304-310. doi: 10.1123/ijspp.2019-0965

Reynés, B., van Schothorst, E. M., Keijer, J., Palou, A., and Oliver, P. (2019). Effects of cold exposure revealed by global transcriptomic analysis in ferret peripheral blood mononuclear cells. Sci. Rep. 9:19985. doi: 10.1038/s41598-019-56354-6

Roberts, L. A., Raastad, T., Markworth, J. F., Figueiredo, V. C., Egner, I. M., Shield, A., et al. (2015). Post-exercise cold water immersion attenuates acute anabolic signalling and long-term adaptations in muscle to strength training. J. Physiol. 593, 4285-4301. doi: 10.1113/JP270570

Sapega, A. A., and Drillings, G. (1983). The definition and assessment of muscular power. J. Orthop. Sports Phys. Ther. 5, 7-9. doi: 10.2519/jospt.1983.5.1.7

Tanabe, Y., Esaki, K., and Nomura, T. (1986). Skeletal muscle pathology in X chromosome-linked muscular dystrophy (mdx) mouse. Acta Neuropathol. 69, 91-95. doi: 10.1007/BF00687043

Tidball, J. G. (2011). Mechanisms of muscle injury, repair, and regeneration. Compr. Physiol. 1, 2029-2062. doi: 10.1002/cphy.c100092

Warren, G. L., Ingalls, C. P., Lowe, D. A., and Armstrong, R. B. (2001). Excitation-contraction uncoupling: major role in contraction-induced muscle injury. Exerc. Sport Sci. Rev. 29, 82-87. doi: 10.1249/00003677-20010400 0-00008

Warren, G. L., Ingalls, C. P., Lowe, D. A., and Armstrong, R. B. (2002). What mechanisms contribute to the strength loss that occurs during and in the recovery from skeletal muscle injury? J. Orthop. Sports Phys. Ther. 32, 58-64. doi: 10.2519/jospt.2002.32.2.58

White, G. E., Rhind, S. G., and Wells, G. D. (2014). The effect of various coldwater immersion protocols on exercise-induced inflammatory response and functional recovery from high-intensity sprint exercise. Eur. J. Appl. Physiol. 114, 2353-2367. doi: 10.1007/s00421-014-2954-2

Winter, E. M., Abt, G., Brookes, F. B. C., Challis, J. H., Fowler, N. E., Knudson, D. V., et al. (2016). Misuse of "Power" and other mechanical terms in sport and exercise science research. J. Strength Cond. Res. 30, 292-300. doi: 10.1519/JSC.0000000000001101

Yamane, M., Ohnishi, N., and Matsumoto, T. (2015). Does regular post-exercise cold application attenuate trained muscle adaptation? Int. J. Sports Med. 36, 647-653. doi: 10.1055/s-0034-1398652

Conflict of Interest: The authors declare that the research was conducted in the absence of any commercial or financial relationships that could be construed as a potential conflict of interest.

Copyright (c) 2021 Lindsay and Peake. This is an open-access article distributed under the terms of the Creative Commons Attribution License (CC BY). The use, distribution or reproduction in other forums is permitted, provided the original author(s) and the copyright owner(s) are credited and that the original publication in this journal is cited, in accordance with accepted academic practice. No use, distribution or reproduction is permitted which does not comply with these terms. 